\title{
Carotenoids Assist in Cyanobacterial Photosystem II Assembly and Function
}

\author{
Tomas Zakar, Hajnalka Laczko-Dobos, Tunde N. Toth and Zoltan Gombos* \\ Laboratory of Plant Lipid Function and Structure, Institute of Plant Biology, Biological Research Centre, Hungarian Academy \\ of Sciences, Szeged, Hungary
}

Carotenoids (carotenes and xanthophylls) are ubiquitous constituents of living organisms. They are protective agents against oxidative stresses and serve as modulators of membrane microviscosity. As antioxidants they can protect photosynthetic organisms from free radicals like reactive oxygen species that originate from water splitting, the first step of photosynthesis. We summarize the structural and functional roles of carotenoids in connection with cyanobacterial Photosystem II. Although carotenoids are hydrophobic molecules, their complexes with proteins also allow cytoplasmic localization. In cyanobacterial cells such complexes are called orange carotenoid proteins, and they protect Photosystem II and Photosystem I by preventing their overexcitation through phycobilisomes (PBS). Recently it has been observed that carotenoids are not only required for the proper functioning, but also for the structural stability of PBSs.

\section{OPEN ACCESS}

Edited by:

Julian Eaton-Rye,

University of Otago, New Zealand

Reviewed by:

Shigeru ltoh,

Nagoya University, Japan Kinga Klodawska,

Jagiellonian University, Poland

*Correspondence:

Zoltan Gombos

gombos.zoltan@gmail.com

Specialty section:

This article was submitted to

Plant Cell Biology,

a section of the journal

Frontiers in Plant Science

Received: 21 October 2015

Accepted: 24 February 2016

Published: 10 March 2016

Citation:

Zakar T, Laczko-Dobos H, Toth TN and Gombos Z (2016) Carotenoids Assist in Cyanobacterial Photosystem

$1 /$ Assembly and Function.

Front. Plant Sci. 7:295.

doi: 10.3389/fpls.2016.00295
Keywords: carotenoids, Photosystem II, cyanobacteria, Synechocystis, phycobilisomes, xanthophylls

\section{ROLES OF CAROTENOIDS IN MODULATING PHOTOSYNTHETIC AND CYTOPLASMIC MEMBRANES OF CYANOBACTERIA}

\section{Carotenoids as Protective Agents}

Carotenoids are a group of pigments that play multiple structural and functional roles. They are molecules with conjugated double bonds, which allow them to participate in photosynthetic functions (Gruszecki and Strzalka, 2005). In cyanobacteria the most abundant carotenoids are carotenes (e.g., $\beta$-carotene) and various types of xanthophylls (synechoxanthin, canthaxanthin, caloxanthin, echinenone, myxoxanthophyll, nostoxanthin, zeaxanthin), which are oxygenated derivates of the carotenes (Takaichi and Mochimaru, 2007; Domonkos et al., 2013; Kusama et al., 2015; Toth et al., 2015).

Carotene and xanthophyll molecules are indispensable components of both cytoplasmic and thylakoidal membranes of cyanobacteria. However, $\beta$-carotene is the only carotenoid, which was localized in PSII by X-ray crystallography (Loll et al., 2005; Umena et al., 2011). As an extremly hydrophobic molecule, $\beta$-carotene forms complexes with proteins, thereby functioning as a bridge between various proteins involved in photosynthetic processes. In thylakoid membranes a protective role of $\beta$-carotene was demonstrated as a scavenger of singlet oxygen (Packer et al., 1981).

Abbreviations: CP43, chlorophyll-protein complex 43; CP47, chlorophyll-protein complex 47; FRP, fluorescence recovery protein; NPQ, non-photochemical quenching; OCP, photoactive orange carotenoid protein; PBS, phycobilisome; PSI and PSII, Photosystem I and II; RC47, reaction center 47; ROS, reactive oxygen species; Synechocystis, Synechocystis sp. PCC6803. 
In contrast to $\beta$-carotene, xanthophylls have not been localized unambiguously. Their presence in the photosystems is expected, and has been demonstrated by various biophysical and biochemical methods (Van der Weij-de Wit et al., 2007; Domonkos et al., 2009). Nevertheless the binding of xanthophylls to membrane proteins has not yet been demonstrated. In the membranes they are positioned in a way that their hydrophilic part faces the aqueous phase.

Free carotenes and xanthophylls occupy the hydrophobic region of membrane bilayers. Accordingly, it was predicted that xanthophylls rigidify membranes, whereas $\beta$-carotene and echinenon have a neutral or fluidizing effect (Gruszecki and Strzalka, 2005).

Although the scavenging character of xanthophylls is stronger than that of $\beta$-carotene (Steiger et al., 1999; Domonkos et al., 2013), neither their localization nor their exact scavenging role has been elucidated yet. Xanthophylls are found mainly in cytoplasmic membranes (Masamoto and Furukawa, 1997; Masamoto et al., 1999). A recent review has summarized the protective mechanism of carotenoids and other putative protective functions against ROS, which are produced by PSII of photosynthesis that mediates light-driven oxidation of water and the release of molecular oxygen (Derks et al., 2015).

\section{Carotenes and Xanthophylls Affect Membrane Viscosity}

Due to their influence on membrane properties, the xanthopylls are also important in the adaptation to various temperature conditions. Low growth temperatures induce enhanced synthesis of xanthophylls and, consequently, the xanthophyll content of thylakoid membranes is increased to compensate for elevated lipid desaturation. Instead of increasing membrane microviscosity, enhanced xanthophyll content alters membrane dynamics by allowing a tighter arrangement of fatty acyl moieties. The observed discrepancy could be explained by an apparent increase of very rigid, myxoxanthophyll-related lipids in the thylakoid membranes (Varkonyi et al., 2002).

\section{Presence of Enzymes of Carotenoid Biosynthesis in Cytoplasmic Membrane}

More recent research has shown that cytoplasmic membranes contain some biosynthetic enzymes specific for the biosynthesis of echinenone and $\beta$-carotene. The CrtQ (zeta-carotene desaturase) and $\mathrm{CrtO}$ (beta-carotene ketolase) enzymes involved in carotenoid synthesis are localized in cytoplasmic membranes (Zhang et al., 2015). This would mean that echinenone and precursors of $\beta$-carotene are more abundant in cytoplasmic membranes than in thylakoids. Cytoplasmic membranes contain higher amounts of carotenoids than thylakoid membranes, which results in a more pronounced modulation of their membrane fluidity.

Despite the relatively low carotenoid content of the thylakoid membrane, these compounds are indispensable for photosynthetic processes. They not only affect the membrane structure, but also influence the oligomerization of proteins, thereby modulating photosynthetic functions.

\section{THE EFFECT OF CAROTENOIDS ON THE ARCHITECTURE AND VARIOUS FUNCTIONS OF PHOTOSYSTEM II COMPLEXES}

\section{Structure}

$\mathrm{X}$-ray crystallography revealed that in thylakoid membranes the main carotene ( $\beta$-carotene) localizes in the photosynthetic reaction centers (Loll et al., 2005; Umena et al., 2011). These results revealed that a PSII complex contains $11 \beta$-carotene molecules. Xanthophylls could not be localized unambiguously. The importance of carotenoids in maintaining the stability and functioning of PSII was demonstrated in a carotenoid-deficient mutant strain (Sozer et al., 2010).

The completely carotenoid deficient $\Delta c r t H / B$ or $\Delta c r t B$ mutants of Synechocystis sp. PCC 6803 (hereafter Synechocystis) are devoid of active PSII reaction centers. These strains do not show oxygen-evolving activity (Sozer et al., 2010; Toth et al., 2015). The cells have reduced translation for a number of genes encoding PSII proteins and hence do not assemble active PSII complexes. They are capable of synthesizing only a limited amount of CP47, which is crucial for the formation of dimeric PSII complexes, but CP43 synthesis is almost completely blocked. The lack of these protein subunits prevents the formation of functional PSII complexes. This is the reason why we think that carotenoids are indispensable components of PSII.

Complete loss of carotenoids prevents the proper assembly of PSII (Figure 1), and such cells contain almost exclusively CP43less oligomers. They show reduced PSII fluorescence, which originates from PSII components that are unable to integrate into a fully functional complex. In the carotenoid-less mutant $\Delta c r t B$ we observed the accumulation of fluorescence-emitting spots with long lifetime close to the cell walls (Toth et al., 2015, Supplementary Figures). This could indicate that PSII biogenesis is blocked and incomplete adducts remain localized at the thylakoid-organizing complexes. This is in good agreement with an earlier observation of dysfunctional thylakoid membrane systems in a carotenoid-deficient mutant (Nickelsen et al., 2011). The PSII structure of Thermosynechococcus elongatus, deduced from X-ray crystallographic data (Guskov et al., 2009) shows the presence of various protein subunits and cofactors, such as carotenoids, in the dimeric form of reaction centers (Figure 1A). The structure of the imagined carotenoid-less PSII is presented in Figure 1B. However, the reaction center in $\Delta$ crtB cells contains almost exclusively CP43-less oligomers. Radioactive labeling demonstrated limited synthesis of inner PSII antennae, CP47 and, particularly, CP43. The lack of CP43 resulted in the formation of the RC47 pre-assembly complex (Komenda et al., 2012), demonstrating that carotenoid deficiency results in low levels of partially assembled reaction center complexes. These cells show reduced fluorescence originating from the remaining chlorophyll-protein complexes that are unable to integrate into a complex (Sozer et al., 2010; Toth et al., 2015).

Carotenoids, together with chlorophylls, are also necessary for the translation and stabilization of photosynthetic reaction center apoproteins in the green alga Chlamydomonas reinhardtii, 


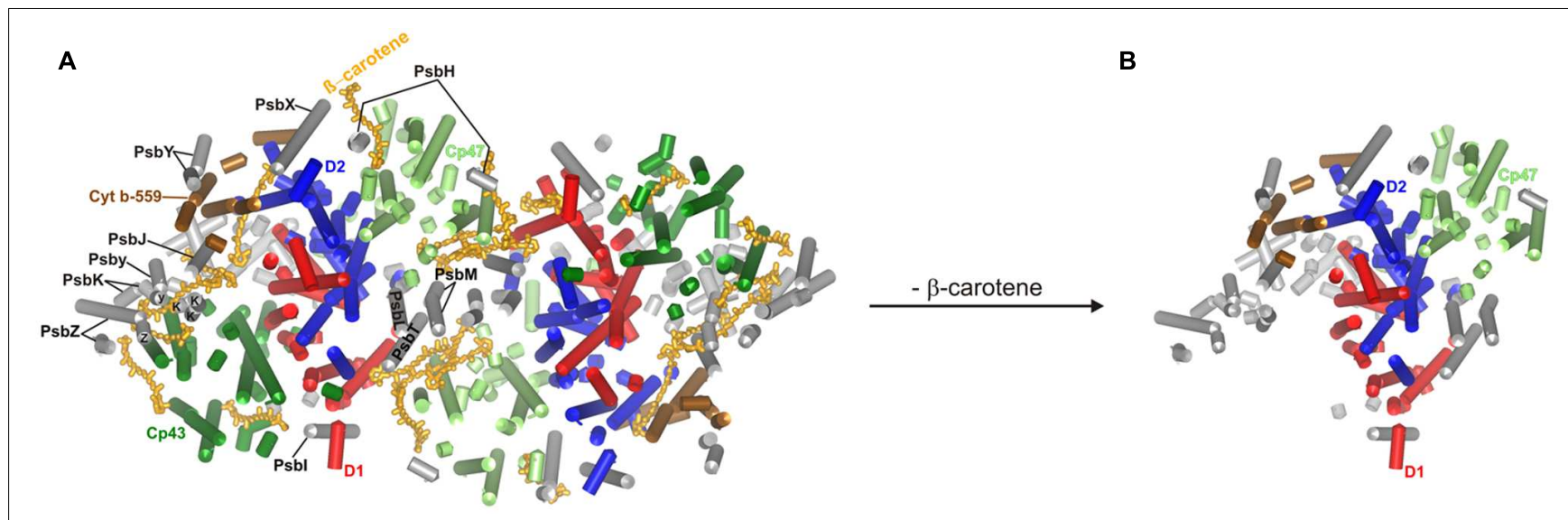

FIGURE 1 | Structure of the PSII complex deduced from X-ray crystallographic data (PDB files: 3BZ1 and 3BZ2). The PSII supercomplex in wild type (A) and assumed PSII structure in carotenoid-deficient (B) Thermosynechococcus elongatus.

however, they do not regulate the transcription of reaction center apoproteins (Herrin et al., 1992).

Our recent results suggest some structural function of xanthophylls in stabilizing PSII, as well as its dimeric complexes in Synechocystis (Toth et al., 2015). Carotenoids are essential mediators of interactions between PSII monomers, highlighting the role of neutral lipids, which can regulate the equilibrium between dimerization and dissociation (Guskov et al., 2009).

Accordingly, carotenoids are indispensable constituents of the photosynthetic apparatus, being essential not only for antioxidative protection but also for the productive synthesis and accumulation of photosynthetic proteins and, especially, those of the PSII antenna subunits.

\section{Function}

Carotenoids, due to their light harvesting (Stamatakis et al., 2014) and photoprotective capacity (Schafer et al., 2005; Sozer et al., 2010), are indispensable for the function of the photosynthetic apparatus, and particularly in that of PSII. Under low light conditions carotenoids can provide more efficient light absorption (Koyama et al., 1996; Bode et al., 2009). By contrast, when cyanobacteria are exposed to high light, excess energy needs to be reduced to avoid photoinhibition damage to the photosystems (Powles, 1984; Aro et al., 1993, 2005). Carotenoids can exert protection by dissipating excess energy as heat, a phenomenon called NPQ, or by scavenging ROS.

The activity of light-damaged PSII is efficiently restored by a repair system, therefore photoinhibition occurs only when the rate of inactivation exceeds that of repair. In order to understand the mechanism by which carotenoids protect the cells under light stress it would be necessary to study the two processes of photoinhibition, photodamage and repair, separately (Nishiyama and Murata, 2014).

Photosystem II complexes are very sensitive not only to strong light but also to a wide range of abiotic stress effects, such as low and high temperatures, UV-B exposure, drought, and high concentration of salts (Takahashi and Murata, 2008). These stress factors ultimately lead to oxidative stress. Recently it has been shown that $\triangle$ sigCDE, a group $2 \sigma$ factor mutant of Synechocystis, is more sensitive to oxidative stress, but also more resistant to the photoinhibition of PSII. In this mutant an up-regulation of photoprotective carotenoids was observed, but it has been suggested that the resistance to light damage of PSII and the overall tolerance to oxidative stress are distinct in cyanobacteria, and their mechanisms are different (Hakkila et al., 2014).

In order to study the specific role of different carotenoids in cyanobacteria a wide range of carotenoid-deficient mutants were generated. Studies with various xanthophyll mutants of Synechococcus sp. PCC 7002 indicate that xanthophylls contribute to protection against photo-oxidative stress (Zhu et al., 2010). Similarly Synechocystis mutants lacking almost all xanthophylls $(\Delta c r t R O)$ were sensitized to photodamage only under high light conditions (Schafer et al., 2005), whereas under normal illumination charge separation in PSII seemed unaffected (Toth et al., 2015). Recently it has been shown that zeaxanthin and echinenon protect the repair part of the PSII recovery cycle from photoinhibition by decreasing the level of singlet oxygen that inhibits protein synthesis (Kusama et al., 2015).

The removal of all $\beta$-carotene and xanthophylls from Synechocystis $(\Delta c r t H / B$ and $\Delta c r t B$ mutants) causes more severe effects. These strains are extremely light sensitive and they can grow only in the dark under light-activated heterotrophic conditions, without detectable oxygen evolution (Sozer et al., 2010). Time-resolved fluorescence (streak camera) measurements of these mutant cells also indicate inactive PSII (Toth et al., 2015).

In PSII reaction centers there are at least two redox active $\beta$-carotenes (Telfer et al., 2003). Recently it has been shown that one of the two so-called redox active carotenoids, $\mathrm{Car}_{\mathrm{D} 2}$, plays a role in photoprotection. Site-directed mutations around the binding pocket of $\mathrm{Car}_{\mathrm{D} 2}$ in Synechocystis cells revealed the importance of $\beta$-carotenes in the initiation of secondary electron transfer processes, which occurs when water oxidation is inhibited (Shinopoulos et al., 2014). 
In addition to the role of carotenoids as constituents of membrane-embedded PSII, they also have crucial functions in the formation of protein complexes, the so-called photoactive orange carotenoid proteins. A recent study has elucidated the dual role of carotenoids in OCP, namely the protection of photosystems by quenching excess energy, and also the capacity of quenching singlet oxygen formed during the light reactions (Sedoud et al., 2014). Using an OCP-deficient mutant it has been shown that OCP-related thermal dissipation protects the repair of PSII during photoinhibition (Kusama et al., 2015). Although it is clear that carotenoids are the principal actors in energy dissipation, the mechanism of this process is poorly understood. Staleva et al. (2015) pointed out a new function of carotenoids associated with proteins. They have shown that Synechocystis HliD, a Hlips (high light-inducible proteins) family protein, binds chlorophyll $a$ and $\beta$-carotene with a 3:1 ratio. The photoprotective role of this carotenoid-binding protein was demonstrated by femtosecond spectroscopy, showing that energy dissipation is achieved via direct energy transfer from a chlorophyll $a$ Qy state to the $\beta$-carotene S1 state (Staleva et al., 2015).

Currently global changes in the environment can generate a multitude of stress factors, such as high or low temperatures, high light or UV-B radiation, etc. This underlines the importance of the evolutionary role of carotenoids and the mechanisms by which they can contribute to the survival of various organisms under extreme conditions.

\section{HOW DO CAROTENOIDS AFFECT THE STRUCTURE AND PROCESSES OF PHYCOBILISOMES OF CYANOBACTERIAL PHOTOSYSTEM II COMPLEXES?}

\section{Structure}

Cyanobacteria have special light-harvesting complexes, PBSs, which can absorb light in a wide spectral range. In addition to PBS, carotenoids can function as accessory pigments to widen the range of absorption. PBSs are comprised of rods attached to a core complex that is directly linked to PSII. In Synechocystis the rods contain phycocyanin, which can harvest long wavelength light and transfer its energy to the allophycocynin core, which then transduces it directly to the PSII reaction center (Maccoll and Guard-Friar, 1986). In the reaction center the main pigment is chlorophyll $a$, whereas in the PBS the chromophore is phycocyanobilin or some other, structurally related pigment.

In the PBS structure the presence of $\beta$-carotene or xanthophylls has not yet been demonstrated. Surprisingly, in carotenoid-less $\Delta c r t B$ cells a large amount of unconnected phycocyanin units has been observed (Toth et al., 2015). Density gradient centrifugation revealed that most of the assembled core complexes had shorter rods than those of the wild type. In this mutant PSI is in monomeric form, only a negligible amount of PSII is formed, and CP43 is mostly detached from PSII. Schematic structures of the photosynthetic complexes, as well as images of the sucrose density gradient-separated PBS components of wild-type Synechocystis and its carotenoid-less $\Delta c r t B$ mutant are shown in Figure 2.

It seems that carotenoids are required for the assembly or maintenance of the complete PBS structure (Toth et al., 2015), suggesting a direct or indirect effect of carotenoids on the structure and functions of this complex (Toth et al., 2015).

\section{Light-Harvesting Process}

Cyanobacterial carotenoid-proteins play an important role in photoprotection (Kerfeld et al., 2003; Kerfeld, 2004). One of these, the water-soluble OCP, has been structurally characterized and has recently emerged as a key player in cyanobacterial photoprotection (Sedoud et al., 2014). OCP was first described by David Krogmann more than 25 years ago (Holt and Krogmann, 1981). Highly conserved homologs of the gene encoding the $34 \mathrm{kDa}$ OCP are present in most of the known cyanobacterial genomes.

The carotenoid composition of the OCP isolated from wildtype cells is as follows: $60 \%$ echinenone, $30 \%$ keto-carotenoid

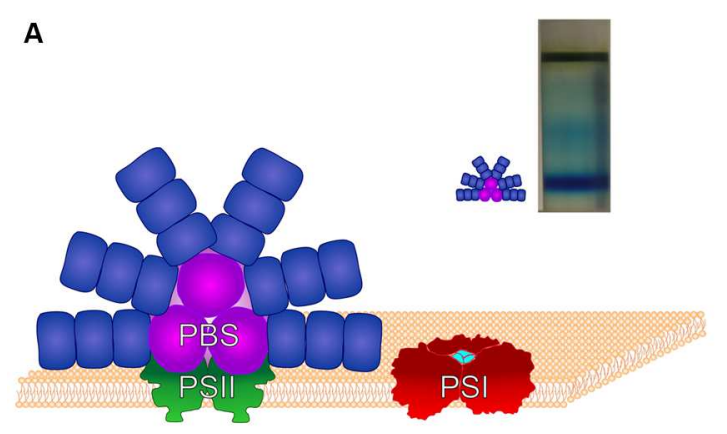

B

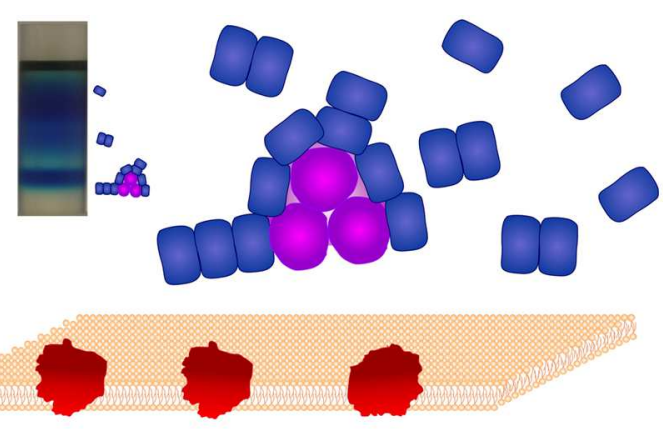

FIGURE 2 | The structures of PBS and the photosynthetic reaction centers in wild-type Synechocystis and its $\Delta$ crtB carotenoid-less mutant. Schematic figure showing the assembled supercomplexes and the positions of their constituents following separation by stepwise sucrose density gradient centrifugation. (A): Fully assembled functional supercomplexes of wild-type cells with entire PBS, dimeric PSII and trimerized PSI. (B): Monomeric PSI and partially assembled PBS of the $\triangle$ crtB mutant. In these cells only a negligible amount of PSII is formed. 
3'-hydroxyechinenone, and 10\% zeaxanthin (Sedoud et al., 2014). The energy collected by the PBS is rapidly transferred from rods to the core, and subsequently to membrane-embedded reaction centers of PSII or PSI.

Photoactive orange carotenoid protein is a photoactive protein. Illumination of OCP by strong blue-green light induces changes in its carotenoid, converting the inactive orange dark form (OCPo) into an active red form (OCPr). In OCPo, $3^{\prime}$-hydroxyechinenone is in all-trans configuration. In OCPr, it is also in all-trans configuration, but its apparent conjugation length increases, resulting in a less distorted, more planar structure. Fourier transform infrared spectra showed that these changes in the carotenoid induce conformational changes in the protein, leading to a less rigid helical structure and a compaction of the $\beta$-sheet. These changes in OCP are essential for the induction of the photoprotective mechanism. Only OCPr is capable of binding to the PBSs, inducing fluorescence quenching and the photoprotective mechanism (Wilson et al., 2012).

In contrast to photosynthetic eukaryotes, photoprotection in cyanobacteria is not induced by transthylakoid $\Delta \mathrm{pH}$ or excitation pressure on PSII. Instead, intense blue-green light (400-550 nm) induces quenching of PSII fluorescence that is reversible in minutes, even in the presence of translation inhibitors (El Bissati et al., 2000). Fluorescence spectra and the study of NPQ mechanism in PBS- and PSII-mutants of Synechocystis indicate that this mechanism involves a specific decrease in the fluorescence emission of PBSs, as well as a decrease of energy transfer from PBS to the reaction centers (Scott et al., 2006; Wilson et al., 2006). The site of the quenching appears to be the core of the PBS (Scott et al., 2006; Wilson et al., 2006; Rakhimberdieva et al., 2007).

The action spectrum of PBS fluorescence quenching resembles the absorption spectrum of cyanobacterial carotenoids. In the absence of OCP, strong white or blue-green lightinduced NPQ was completely inhibited in Synechocystis. As a consequence, OCP-deficient cells are more sensitive to light stress. Moreover, the action spectrum of cyanobacterial NPQ (Rakhimberdieva et al., 2004) exactly matches the absorption spectrum of the carotenoid, $3^{\prime}$-hydroxyechinenone (Polivka et al., 2005) in the OCP. OCP is now known to be specifically involved in the PBS -associated NPQ and not in the other mechanisms affecting the levels of fluorescence, such as state transitions or D1 damage (Wilson et al., 2006; Zhang et al., 2014). Electron microscopic studies using immunogold labeling revealed that the majority of OCP is localized in the inter-thylakoidal cytoplasmic region, on the PBS side of the membrane (Wilson et al., 2006). The interaction between the OCP and the PBSs and thylakoids was corroborated by the presence of OCP in the PBSassociated membrane fraction (Wilson et al., 2006, 2007). In Synechocystis, OCP is constitutively expressed, and it is present even in mutants that lack PBSs (Wilson et al., 2007). Stress conditions (high light, salt stress, iron starvation) increase levels of the OCP transcript and proteins (Hihara et al., 2001; Kanesaki et al., 2002; Fulda et al., 2006; Wilson et al., 2007). All known OCP-like genes of cyanobacteria are transcriptionally active and the NPQ mechanism is inducible by blue light. This suggests that the OCP-based photoprotective mechanism is widespread in cyanobacteria (Boulay et al., 2008).

Fluorescence recovery protein is known to be involved in photoprotection and restoration of full light-harvesting capacity. FRP is needed for the recovery of full antenna capacity when light intensity decreases (Boulay et al., 2010). FRP interacts with the activated OCP and accelerates its deactivation and detachment from the PBS (Boulay et al., 2010; Gwizdala et al., 2011), although the details of these processes have not yet been elucidated (Zhang et al., 2014).

Carotenoid containing OCP molecules have an essential role in cyanobacterial photoprotection by binding to the PBS.

\section{CONCLUSION}

Carotenoids are important for multiple PSII functions, as they are not only required for its activity but also participate in the light-harvesting process. They act as pigments, which can increase the spectral range, and can also protect against overexcitation and oxidative side products. It has been shown that $\beta$-carotene is essential for the assembly of PSII and the PSI trimer, whereas xanthophylls can stabilize them. The influence of OCP on the light-harvesting capacity of PBS and the requirement of carotenoids for the proper assembly and function of this complex highlight important additional roles of not only $\beta$-carotene, but also of the xanthophylls in cyanobacterial photosynthesis. Further studies are required to elucidate the exact mechanisms by which carotenoids influence the structure and function of PSII and the PBS, and how they contribute to the protection of the photosynthetic processes.

\section{AUTHOR CONTRIBUTIONS}

The authors of this mini-review equally contributed to its concept and design. The tasks were assigned as follows: TZ contributed to all chapters and had a key role in formulating the review. HL-D wrote a chapter, critically read and corrected the entire manuscript, and adjusted it to the required format. TT prepared the figures, the list of references, and gave advice on the text. ZG provided the general concept and design, and also contributed to a chapter.

\section{ACKNOWLEDGMENTS}

This work was supported by grants K108411 and PD108551 from the Hungarian Scientific Research Fund, and a bilateral project between the science academies of the Czech Republic and Hungary (HU/2013/06). The authors are thankful to Miklós Szekeres for reading and correcting the manuscript and to Mihály Kis for his help in preparing the figures. 


\section{REFERENCES}

Aro, E. M., Suorsa, M., Rokka, A., Allahverdiyeva, Y., Paakkarinen, V., Saleem, A., et al. (2005). Dynamics of photosystem II: a proteomic approach to thylakoid protein complexes. J. Exp. Bot. 56, 347-356. doi: 10.1093/jxb/eri041

Aro, E. M., Virgin, I., and Andersson, B. (1993). Photoinhibition of Photosystem II - Inactivation, protein damage and turnover. Biochim. Biophys. Acta 1143, 113-134. doi: 10.1016/0005-2728(93)90134-2

Bode, S., Quentmeier, C. C., Liao, P. N., Hafi, N., Barros, T., Wilk, L., et al. (2009). On the regulation of photosynthesis by excitonic interactions between carotenoids and chlorophylls. Proc. Natl. Acad. Sci. U.S.A. 106, 12311-12316. doi: 10.1073/pnas.0903536106

Boulay, C., Abasova, L., Six, C., Vass, I., and Kirilovsky, D. (2008). Occurrence and function of the orange carotenoid protein in photoprotective mechanisms in various cyanobacteria. Biochim. Biophys. Acta 1777, 1344-1354. doi: 10.1016/j.bbabio.2008.07.002

Boulay, C., Wilson, A., D'haene, S., and Kirilovsky, D. (2010). Identification of a protein required for recovery of full antenna capacity in OCP-related photoprotective mechanism in cyanobacteria. Proc. Natl. Acad. Sci. U.S.A. 107, 11620-11625. doi: 10.1073/pnas.1002912107

Derks, A., Schaven, K., and Bruce, D. (2015). Diverse mechanisms for photoprotection in photosynthesis. Dynamic regulation of photosystem II excitation in response to rapid environmental change. Biochim. Biophys. Acta 1847, 468-485. doi: 10.1016/j.bbabio.2015.02.008

Domonkos, I., Kis, M., Gombos, Z., and Ughy, B. (2013). Carotenoids, versatile components of oxygenic photosynthesis. Prog. Lipid Res. 52, 539-561. doi: 10.1016/j.plipres.2013.07.001

Domonkos, I., Malec, P., Laczko-Dobos, H., Sozer, O., Klodawska, K., Wada, H., et al. (2009). Phosphatidylglycerol depletion induces an increase in myxoxanthophyll biosynthetic activity in Synechocystis PCC 6803 Cells. Plant Cell Phys. 50, 374-382. doi: 10.1093/pcp/pcn204

El Bissati, K., Delphin, E., Murata, N., Etienne, A. L., and Kirilovsky, D. (2000). Photosystem II fluorescence quenching in the cyanobacterium Synechocystis PCC 6803: involvement of two different mechanisms. Biochim. Biophys. Acta 1457, 229-242. doi: 10.1016/S0005-2728(00)00104-3

Fulda, S., Mikkat, S., Huang, F., Huckauf, J., Marin, K., Norling, B., et al. (2006). Proteome analysis of salt stress response in the cyanobacterium Synechocystis sp. strain PCC 6803. Proteomics 6, 2733-2745. doi: 10.1002/pmic.200500538

Gruszecki, W. I., and Strzalka, K. (2005). Carotenoids as modulators of lipid membrane physical properties. Biochim. Biophys. Acta 1740, 108-115. doi: 10.1016/j.bbadis.2004.11.015

Guskov, A., Kern, J., Gabdulkhakov, A., Broser, M., Zouni, A., and Saenger, W. (2009). Cyanobacterial photosystem II at 2.9-angstrom resolution and the role of quinones, lipids, channels and chloride. Nat. Struct. Mol. Biol. 16, 334-342. doi: $10.1038 /$ nsmb.1559

Gwizdala, M., Wilson, A., and Kirilovsky, D. (2011). In vitro reconstitution of the cyanobacterial photoprotective mechanism mediated by the orange carotenoid protein in Synechocystis PCC 6803. Plant Cell 23, 2631-2643. doi: 10.1105/tpc.111.086884

Hakkila, K., Antal, T., Rehman, A. U., Kurkela, J., Wada, H., Vass, I., et al. (2014). Oxidative stress and photoinhibition can be separated in the cyanobacterium Synechocystis sp. PCC 6803. Biochim. Biophys. Acta 1837, 217-225. doi: 10.1016/j.bbabio.2013.11.011

Herrin, D. L., Battey, J. F., Greer, K., and Schmidt, G. W. (1992). Regulation of chlorophyll apoprotein expression and accumulation - Requirements for carotenoids and chlorophyll. J. Biol. Chem. 267, 8260-8269.

Hihara, Y., Kamei, A., Kanehisa, M., Kaplan, A., and Ikeuchi, M. (2001). DNA microarray analysis of cyanobacterial gene expression during acclimation to high light. Plant Cell 13, 793-806. doi: 10.1105/tpc.13.4.793

Holt, T. K., and Krogmann, D. W. (1981). A carotenoid-protein from cyanobacteria. Biochim. Biophys. Acta 637, 408-414. doi: 10.1016/00052728(81)90045-1

Kanesaki, Y., Suzuki, I., Allakhverdiev, S. I., Mikami, K., and Murata, N. (2002). Salt stress and hyperosmotic stress regulate the expression of different sets of genes in Synechocystis sp. PCC 6803. Biochem. Biophys. Res. Commun. 290, 339-348. doi: $10.1006 /$ bbrc.2001.6201

Kerfeld, C. A. (2004). Water-soluble carotenoid proteins of cyanobacteria. Arch. Biochem. Biophys. 430, 2-9. doi: 10.1016/j.abb.2004.03.018
Kerfeld, C. A., Sawaya, M. R., Brahmandam, V., Cascio, D., Ho, K. K., TrevithickSutton, C. C., et al. (2003). The crystal structure of a cyanobacterial watersoluble carotenoid binding protein. Structure 11, 55-65. doi: 10.1016/S09692126(02)00936-X

Komenda, J., Sobotka, R., and Nixon, P. J. (2012). Assembling and maintaining the photosystem II complex in chloroplasts and cyanobacteria. Curr. Opin. Plant Biol. 15, 245-251. doi: 10.1016/j.pbi.2012.01.017

Koyama, Y., Kuki, M., Andersson, P. O., and Gillbro, T. (1996). Singlet excited states and the light-harvesting function of carotenoids in bacterial photosynthesis. Photochem. Photobiol. 63, 243-256. doi: 10.1111/j.17511097.1996.tb03021.x

Kusama, Y., Inoue, S., Jimbo, H., Takaichi, S., Sonoike, K., Hihara, Y., et al. (2015). Zeaxanthin and echinenone protect the repair of photosystem II from inhibition by singlet oxygen in Synechocystis sp. PCC 6803. Plant Cell Physiol. 56, 906-916. doi: 10.1093/pcp/pcv018

Loll, B., Kern, J., Saenger, W., Zouni, A., and Biesiadka, J. (2005). Towards complete cofactor arrangement in the 3.0 angstrom resolution structure of photosystem II. Nature 438, 1040-1044. doi: 10.1038/nature04224

Maccoll, R., and Guard-Friar, D. (1986). Phycobiliproteins. Boca Raton, FL: CRC Press.

Masamoto, K., and Furukawa, K. (1997). Accumulation of zeaxanthin in cells of the cyanobacterium, Synechococcus sp. strain PCC 7942 grown under high irradiance. J. Plant Physiol. 151, 257-261. doi: 10.1093/pcp/pcu199

Masamoto, K., Zsiros, O., and Gombos, Z. (1999). Accumulation of zeaxanthin in cytoplasmic membranes of the cyanobacterium Synechococcus sp. strain PCC 7942 grown under high light condition. J. Plant Physiol. 155, 136-138. doi: 10.1016/S0176-1617(99)80155-2

Nickelsen, J., Rengstl, B., Stengel, A., Schottkowski, M., Soll, J., and Ankele, E. (2011). Biogenesis of the cyanobacterial thylakoid membrane system an update. FEMS Microbiol. Lett. 315, 1-5. doi: 10.1111/j.1574-6968.2010. 02096.X

Nishiyama, Y., and Murata, N. (2014). Revised scheme for the mechanism of photoinhibition and its application to enhance the abiotic stress tolerance of the photosynthetic machinery. Appl. Microbiol. Biotechnol. 98, 8777-8796. doi: 10.1007/s00253-014-6020-0

Packer, J. E., Mahood, J. S., Mora-Arellano, V. O., Slater, T. F., Willson, R. L., and Wolfenden, B. S. (1981). Free radicals and singlet oxygen scavengers: reaction of a peroxy-radical with beta-carotene, diphenyl furan and 1,4diazobicyclo $(2,2,2)$-octane. Biochem. Biophys. Res. Commun. 98, 901-906. doi: 10.1016/0006-291X(81)91196-7

Polivka, T., Kerfeld, C. A., Pascher, T., and Sundstrom, V. (2005). Spectroscopic properties of the carotenoid $3^{\prime}$-hydroxyechinenone in the orange carotenoid protein from the cyanobacterium Arthrospira maxima. Biochemistry 44, 39944003. doi: 10.1021/bi047473t

Powles, S. B. (1984). Photoinhibition of photosynthesis induced by visible-light. Annu. Rev. Plant Physiol. Plant Mol. Biol. 35, 15-44. doi: 10.1146/annurev.pp.35.060184.000311

Rakhimberdieva, M. G., Stadnichuk, I. N., Elanskaya, T. V., and Karapetyan, N. V. (2004). Carotenoid-induced quenching of the phycobilisome fluorescence in photosystem II-deficient mutant of Synechocystis sp. FEBS Lett. 574, 85-88. doi: 10.1016/j.febslet.2004.07.087

Rakhimberdieva, M. G., Vavilin, D. V., Vermaas, W. F., Elanskaya, I. V., and Karapetyan, N. V. (2007). Phycobilin/chlorophyll excitation equilibration upon carotenoid-induced non-photochemical fluorescence quenching in phycobilisomes of the Synechocystis sp. PCC 6803. Biochim. Biophys. Acta 1767, 757-765. doi: 10.1016/j.bbabio.2006.12.007

Schafer, L., Vioque, A., and Sandmann, G. (2005). Functional in situ evaluation of photo synthesis-protecting carotenoids in mutants of the cyanobacterium Synechocystis PCC 6803. J. Photochem. Photobiol. B Biol. 78, 195-201. doi: 10.1016/j.jphotobiol.2004.11.007

Scott, M., Mccollum, C., Vasilev, S., Crozier, C., Espie, G. S., Krol, M., et al. (2006). Mechanism of the down regulation of photosynthesis by blue light in the cyanobacterium Synechocystis sp. PCC 6803. Biochemistry 45, 8952-8958. doi: $10.1021 /$ bi060767p

Sedoud, A., Lopez-Igual, R., Rehman, A. U., Wilson, A., Perreau, F., Boulay, C., et al. (2014). The cyanobacterial photoactive orange carotenoid protein is an excellent singlet oxygen quencher. Plant Cell 26, 1781-1791. doi: $10.1105 /$ tpc. 114.123802 
Shinopoulos, K. E., Yu, J., Nixon, P. J., and Brudvig, G. W. (2014). Using sitedirected mutagenesis to probe the role of the D2 carotenoid in the secondary electron-transfer pathway of photosystem II. Photosynth. Res. 120, 141-152. doi: 10.1007/s11120-013-9793-6

Sozer, O., Komenda, J., Ughy, B., Domonkos, I., Laczko-Dobos, H., Malec, P., et al. (2010). Involvement of carotenoids in the synthesis and assembly of protein subunits of photosynthetic reaction centers of Synechocystis sp. PCC 6803. Plant Cell Physiol. 51, 823-835. doi: 10.1093/pcp/pcq031

Staleva, H., Komenda, J., Shukla, M. K., Slouf, V., Kana, R., Polivka, T., et al. (2015). Mechanism of photoprotection in the cyanobacterial ancestor of plant antenna proteins. Nat. Chem. Biol. 11, 287-U296. doi: 10.1038/Nchembio.1755

Stamatakis, K., Tsimilli-Michael, M., and Papageorgiou, G. C. (2014). On the question of the light-harvesting role of beta-carotene in photosystem II and photosystem I core complexes. Plant Physiol. Biochem. 81, 121-127. doi: 10.1016/j.plaphy.2014.01.014

Steiger, S., Schafer, L., and Sandmann, G. (1999). High-light-dependent upregulation of carotenoids and their antioxidative properties in the cyanobacterium Synechocystis PCC 6803. J. Photochem. Photobiol. B Biol. 52, 14-18. doi: 10.1016/S1011-1344(99)00094-9

Takahashi, S., and Murata, N. (2008). How do environmental stresses accelerate photoinhibition? Trends Plant Sci. 13, 178-182. doi: 10.1016/ j.tplants.2008.01.005

Takaichi, S., and Mochimaru, M. (2007). Carotenoids and carotenogenesis in cyanobacteria: unique ketocarotenoids and carotenoid glycosides. Cell. Mol. Life Sci. 64, 2607-2619. doi: 10.1007/s00018-007-7190-Z

Telfer, A., Frolov, D., Barber, J., Robert, B., and Pascal, A. (2003). Oxidation of the two beta-carotene molecules in the photosystem II reaction center. Biochemistry 42, 1008-1015. doi: 10.1021/bi026206p

Toth, T. N., Chukhutsina, V., Domonkos, I., Knoppova, J., Komenda, J., Kis, M., et al. (2015). Carotenoids are essential for the assembly of cyanobacterial photosynthetic complexes. Biochim. Biophys. Acta 1847, 1153-1165. doi: 10.1016/j.bbabio.2015.05.020

Umena, Y., Kawakami, K., Shen, J. R., and Kamiya, N. (2011). Crystal structure of oxygen-evolving photosystem II at a resolution of 1.9 angstrom. Nature 473, 55-65. doi: 10.1038/nature09913

Van der Weij-de Wit, C. D., Ihalainen, J. A., Van De Vijver, E., D’haene, S., Matthijs, H. C., Van Grondelle, R., et al. (2007). Fluorescence quenching of IsiA in early stage of iron deficiency and at cryogenic temperatures. Biochim. Biophys. Acta 1767, 1393-1400. doi: 10.1016/j.bbabio.2007.10.001
Varkonyi, Z., Masamoto, K., Debreczeny, M., Zsiros, O., Ughy, B., Gombos, Z., et al. (2002). Low-temperature-induced accumulation of xanthophylls and its structural consequences in the photosynthetic membranes of the cyanobacterium Cylindrospermopsis raciborskii: an FTIR spectroscopic study. Proc. Natl. Acad. Sci. U.S.A. 99, 2410-2415. doi: 10.1073/pnas.042698799

Wilson, A., Ajlani, G., Verbavatz, J. M., Vass, I., Kerfeld, C. A., and Kirilovsky, D. (2006). A soluble carotenoid protein involved in phycobilisomerelated energy dissipation in cyanobacteria. Plant Cell 18, 992-1007. doi: 10.1105/tpc.105.040121

Wilson, A., Boulay, C., Wilde, A., Kerfeld, C. A., and Kirilovsky, D. (2007). Lightinduced energy dissipation in iron-starved cyanobacteria: roles of OCP and IsiA proteins. Plant Cell 19, 656-672. doi: 10.1105/tpc.106.045351

Wilson, A., Gwizdala, M., Mezzetti, A., Alexandre, M., Kerfeld, C. A., and Kirilovsky, D. (2012). The essential role of the N-terminal domain of the orange carotenoid protein in cyanobacterial photoprotection: importance of a positive charge for phycobilisome binding. Plant Cell 24, 1972-1983. doi: 10.1105/tpc.112.096909

Zhang, H., Liu, H., Niedzwiedzki, D. M., Prado, M., Jiang, J., Gross, M. L., et al. (2014). Molecular mechanism of photoactivation and structural location of the cyanobacterial orange carotenoid protein. Biochemistry 53, 13-19. doi: 10.1021/bi401539w

Zhang, L., Selao, T. T., Selstam, E., and Norling, B. (2015). Subcellular localization of carotenoid biosynthesis in Synechocystis sp. PCC 6803. PLoS ONE 10:e0130904. doi: 10.1371/journal.pone.0130904

Zhu, Y., Graham, J. E., Ludwig, M., Xiong, W., Alvey, R. M., Shen, G., et al. (2010). Roles of xanthophyll carotenoids in protection against photoinhibition and oxidative stress in the cyanobacterium Synechococcus sp. strain PCC 7002. Arch. Biochem. Biophys. 504, 86-99. doi: 10.1016/j.abb.2010.07.007

Conflict of Interest Statement: The authors declare that the research was conducted in the absence of any commercial or financial relationships that could be construed as a potential conflict of interest.

Copyright (c) 2016 Zakar, Laczko-Dobos, Toth and Gombos. This is an open-access article distributed under the terms of the Creative Commons Attribution License (CC BY). The use, distribution or reproduction in other forums is permitted, provided the original author(s) or licensor are credited and that the original publication in this journal is cited, in accordance with accepted academic practice. No use, distribution or reproduction is permitted which does not comply with these terms. 